Ann. Biol. anim. Bioch. Biophys., 1979, 19 (2 A), 405-412.

\title{
Effet d'une interruption et de restitutions partielles du recyclage entéro-hépatique sur le niveau de l'excrétion biliaire chez le porc
}

\author{
par Catherine JUSTE, T. CORRING \\ avec la collaboration technique de Geargette FREDOU, A. ROGER et A. VAN HECKE \\ Laboratoire de Physiologie de la Nutrition \\ I.N.R.A.-C.N.R.Z., 78350 jouy en Josas.
}

Summary. Effect of interruption and of partial restitution of the enterohepatic cycle upon bile excretion in pigs.

The effect of interruption and of partial restitution of the enterohepatic cycle (EHC) upon bile excretion was studied in 9 fistulated pigs. In normal EHC, bile excretion remained constant in the same animal during the 8 to 10 days of observation. Bile volume and total bile salts excreted in $24 \mathrm{hrs}$. were, on the average, $2053 \mathrm{ml}$ and $42.7 \mathrm{~g}$, respectively. The interruption of the EHC led to an immediate and marked decrease of the total bile salts excreted; the level reached represented about 20 p. 100 of the initial value and did not significantly vary throughout the period of the EHC interruption (2 to 11 days). Bile volumes were less affected since they decreased to a level which was about 80 p. 100 of the initial value. Partial restitution of the EHC in 2 pigs showed that bile salt excretion was always higher than the level of bile salt restitution. Such a compensation was higher with the lower restitution level.

\section{Introduction.}

Chez l'Homme (Hardison et Rosenberg, 1967 ; Mc Leod et Wiggins, 1968 ; Meihoff et Kern, 1968 ; Hoffman et Poley, 1972) une interruption du recyclage entérohépatique biliaire après résection iléale, conduit à une déficience plus ou moins sévère en sels biliaires dans la lumière intestinale. Cependant les résultats, obtenus soit par drainage duodénal soit par analyse fécale, ne renseignent pas sur la quantifé de bile et de sels biliaires parvenant à l'intestin. Or on connaît le rôle essentiel des sels biliaires dans l'hydrolyse et l'absorption des lipides alimentaires. II nous est apparu importanf d'étudier expérimentalement les effets d'une interruption ou de restitutions partielles du recyclage entérohépatique sur le niveau de l'excrétion biliaire pour mieux appréhen- 
der les mécanismes impliqués dans la digestion défectueuse et la malabsorption lipidiques induites.

Une telle étude a été réalisée chez le Singe par Dowling, Mack et Small (1970) et nous proposons d'utiliser comme modèle expérimental le Porc dont la physiologie digestive et les modalités physico-chimiques de la lipolyse en particulier, sont semblables à celles de l'Homme.

\section{Matériel et méthodes.}

Animaux ef techniques. - 9 porcs mâles castrés, de race Large White et de poids vif compris entre 40 et $50 \mathrm{~kg}$, ont été munis d'une fistule cholédocienne et d'une fistule duodénale. La technique opératoire, le mode de contention et le régime alimentaire (2,11 p. 100 de lipides et 16,86 p. 100 de protéines) sont identiques à ceux utilisés dans un précédent travail (Juste, Corring et Breant, 1979).

Le volume de bile recueillie est relevé 3 fois par jour (à 9 h, 13 h et 17 h). Un prélèvement de 5 à $10 \mathrm{ml}$ est effectué lors de chaque relevé, de telle sorte que les échantillonnages journaliers ne dépassent pas 1 à 2 p. 100 du volume total excrété par 24 heures. Chaque échantillon est soumis à un dosage enzymatique des stéroïdes biliaires avec la $3 \alpha$-hydroxystéroïde-déhydrogénase. Après lecture volumétrique et échantillonnage, la bile est destinée à la réintroduction duodénale à l'aide d'une pompe péristaltique dont le débit calque approximativement le débit cholédocien.

Schéma expérimental. - Après complet rétablissement post-opératoire, soit 10 à 15 jours après l'intervention chirurgicale, les animaux ont été expérimentés pendant une durée moyenne de $13 \pm 2$ jours.

Chaque porc a été soumis à une période d'observation préliminaire de 8 jours en moyenne, visant à déterminer le niveau de l'excrétion biliaire, en volume $(\mathrm{ml} / 24 \mathrm{~h})$, concentration $(\mathrm{mg} / \mathrm{ml})$ et quantité de sels biliaires totaux $(\mathrm{g} / 24 \mathrm{~h})$, dans le cas d'un recyclage entérohépatique normal (restitution duodénale de la totalité de la bile recueillie par écoulement cholédocien).

Puis les animaux (excepté le porc 2) ont éfé soumis à une interruption du recyclage entérohépatique : à $9 \mathrm{~h}$ du matin, la pompe de réintroduction de la bile a été arrêtée. La totalité de la sécrétion a ainsi été dérivée de la lumière intestinale pendant une durée de 2 à 11 jours selon les animaux (tabl. 1). Le niveau journalier de l'excrétion biliaire (en volume, concentration et quantité de sels biliaires) a été enregistré pour chaque animal, en réponse à ce traitement. A la suite de cette dernière période, le recyclage entérohépatique de la bile a été rétabli chez 5 animaux.

Enfin, 2 animaux (porcs 1 et 2) ont été utilisés pour étudier l'effet de restitutions partielles du recyclage entérohépatique sur le niveau de l'excrétion biliaire. Dans ce but, une partie seulement de la bile recueillie a été réintroduite dans le duodénum. La quantité recyclée quotidiennement est exprimée en p. 100 de la quantité de sels biliaires excrétés normalement sur $24 \mathrm{~h}$. Les niveaux de réintroduction et leur durée d'application ont été : 11 p. 100 pendant 3 jours et 25 p. 100 pendant 2 jours, pour le porc 1 , et 7 p. 100 pendant 2 jours, 14 p. 100 pendant 3 jours et 33 p. 100 pendant 4 jours, pour le porc 2 . 
TABLEAU 1

Effet d'une interruption du recyclage entéro-hépatique sur la quantifé de bile excrêtée

\begin{tabular}{cccc}
\hline Porc & $\begin{array}{c}\text { Volumes de bile émis par 24 heures : } \\
\text { lors d'une interruption } \\
\text { du recyclage } \\
\bar{x} \pm S \bar{x}\end{array}$ & $\begin{array}{c}\text { Signification } \\
\text { statistique }\end{array}$ \\
\hline 1 & $\bar{x}\left({ }^{1}\right) \pm S \bar{x}\left({ }^{2}\right)$ & $1273 \pm 47(11)$ & $P<0,001$ \\
3 & $1705 \pm 76(4)\left({ }^{3}\right)$ & $1953 \pm 44(3)$ & $\mathrm{P}<0,01$ \\
4 & $2492 \pm 72(19)$ & $1920 \pm 130(2)$ & $\mathrm{N} . \mathrm{S}$. \\
5 & $2150 \pm 146(9)$ & $1892 \pm 72(6)$ & $\mathrm{P}<0,01$ \\
6 & $2615 \pm 241(4)$ & $1317 \pm 70(5)$ & $\mathrm{P}<0,005$ \\
7 & $1831 \pm 84(8)$ & $1397 \pm 347(3)$ & $\mathrm{N}$. S. \\
8 & $1416 \pm 116(4)$ & $1416 \pm 125(2)$ & $\mathrm{P}<0,001$ \\
9 & $2037 \pm 126(6)$ & $1617 \pm 103(37)$ & $\mathrm{P}<0,05$ \\
\hline moyenne & $2175 \pm 90(8)$ & & \\
\hline
\end{tabular}
(1) : $\bar{x}=$ moyennes exprimées en $\mathrm{ml} / 24 \mathrm{~h}$.
(2) $S \bar{x}=$ écarts types de la moyenne exprimés en $\mathrm{ml} / 24 \mathrm{~h}$.
(3) : nombre de journées d'observation.

\section{Résultats.}

Tous les animaux opérés ont été utilisés. Dans tous les cas, le rejet du cathéter cholédocien a déłerminé l'arrêt de l'expérimentation. Les animaux se sont alimentés normalement pendant toute la durée de l'observation, présentant une croissance journalière de 400 à $600 \mathrm{~g}$. Enfin, les dérivations biliaires n'ont provoqué aucune diarrhée pour le régime alimentaire distribué ; cependant l'observation macroscopique des fèces a laissé supposer l'existence d'une légère stéatorrhée.

\section{TABLEAU 2}

Effet d'une interruption du recyclage entéro-hépatique sur la concentration de la bile en sels biliaires tolaux

\begin{tabular}{cccc}
\hline Porc & $\begin{array}{c}\text { Concentrations en sels biliaires, observées sur } 24 \mathrm{~h}: \\
\text { lors d'une interruption } \\
\text { du recyclage } \\
\overline{\mathbf{x}} \pm \mathrm{S} \overline{\mathrm{x}}\end{array}$ & $\begin{array}{c}\text { Signification } \\
\text { statistique }\end{array}$ \\
\hline $\mathbf{x}\left({ }^{1}\right) \pm \mathrm{S} \overline{\mathrm{x}}\left({ }^{2}\right)$ & $6,4 \pm 0,3(11)$ & $\mathrm{P}<0,001$ \\
3 & $23,7 \pm 3,0(4)\left({ }^{3}\right)$ & $4,3 \pm 0,4(3)$ & $\mathrm{P}<0,001$ \\
4 & $17,2 \pm 0,9(19)$ & $\mathrm{P}<0,001$ \\
5 & $16,2 \pm 0,9(9)$ & $4,1 \pm 0,5(2)$ & $\mathrm{P}<0,001$ \\
6 & $28,0 \pm 2,1(4)$ & $6,1 \pm 0,1(5)$ & $\mathrm{P}<0,005$ \\
7 & $18,3 \pm 2,2(8)$ & $5,6 \pm 0,5(3)$ & $\mathrm{P}<0,005$ \\
8 & $15,2 \pm 0,5(4)$ & $8,1 \pm 3,2(2)$ & $\mathrm{P}<0,001$ \\
9 & $24,5 \pm 1,7(6)$ & $3,5 \pm 0,4(5)$ & $\mathrm{P}<0,001$ \\
\hline moyenne & $20,3 \pm 1,6(8)$ & $4,9 \pm 0,6(37)$ & \\
\hline
\end{tabular}

(1) $\bar{x}:$ moyennes exprimées en $\mathrm{mg} / \mathrm{ml}$.

( $\left.{ }^{2}\right) \mathrm{S} \tilde{X}$ : écarts types de la moyenne exprimés en $\mathrm{mg} / \mathrm{ml}$.

$\left({ }^{3}\right)$ : nombre de journées d'observation. 
Lorsque le recyclage entérohépatique de la bile est conservé, l'excrétion biliaire journalière pour tous les animaux et nycthémères confondus est de $2053 \pm 140 \mathrm{ml}$ de bile et $42,7 \pm 5,2 \mathrm{~g}$ de sels biliaires totaux. Les variations individuelles ef celles observées d'un nycthémère au suivant pour le même animal sont voisines des variations enregistrées précédemment (Juste, Corring et Breant, 1979). La variabilité intraanimale reste généralement inférieure à la variabilité entre animaux (tabl. 1, 2 et 3), ce qui traduit une relative stabilité de l'excrétion biliaire quotidienne chez le même porc.

Niveau de l'excrétion biliaire chez le Porc, dans le cas d'une interruption du recyclage entérohépatique (tabl. 1, 2 et 3).

TABLEAU 3

Effet d'une interruption du recyclage entéro-hépatique sur la quantité de sels biliaires exportés

\begin{tabular}{cccc}
\hline Porc & $\begin{array}{c}\text { Quantités de seis biliaires exportés par } 24 \mathrm{~h} \\
\text { lors d'une interruption } \\
\text { du recyclage } \\
\overline{\mathrm{x}} \pm \mathrm{S} \overline{\mathrm{x}}\end{array}$ & $\begin{array}{c}\text { Signification } \\
\text { statistique }\end{array}$ \\
\hline $\mathbf{1}$ & $\overline{\mathrm{X}}\left({ }^{1}\right) \pm \mathrm{S} \overline{\mathrm{x}}\left({ }^{2}\right)$ & $8,1 \pm 0,5(11)$ & $\mathrm{P}<0,001$ \\
3 & $40,7 \pm 5,9(4)\left({ }^{3}\right)$ & $\mathrm{P}<0,001$ \\
4 & $43,7 \pm 3,3(19)$ & $\mathrm{P}<0,005$ \\
5 & $34,7 \pm 2,7(9)$ & $7,8 \pm 0,6(3)$ & $\mathrm{P}<0,001$ \\
6 & $72,0 \pm 3,6(4)$ & $7,6 \pm 0,7(6)$ & $\mathrm{P}<0,005$ \\
7 & $34,6 \pm 5,6(8)$ & $8,0 \pm 0,5(5)$ & $\mathrm{P}<0,005$ \\
8 & $21,4 \pm 1,2(4)$ & $8,0 \pm 2,3(3)$ & $\mathrm{P}<0,001$ \\
9 & $50,0 \pm 4,8(6)$ & $14,6 \pm 6,7(2)$ & $\mathrm{P}<0,001$ \\
\hline moyenne & $44,2 \pm 4,1(8)$ & $4,9 \pm 0,3(5)$ & \\
\hline
\end{tabular}

(1) : $\bar{x}:$ moyennes exprimées en $g / 24 \mathrm{~h}$.

(2) : $S x$ : écarts types de la moyenne exprimés en $g / 24 \mathrm{~h}$.

(3) : nombre de journées d'observation.

Le niveau de bile recueillie $24 \mathrm{~h}$ après l'interruption du recyclage entérohépatique décroît et atteint $1617 \pm 103 \mathrm{ml}(\mathrm{P}<0,05)$, accusant une diminution de $21 \mathrm{p} .100$ environ par rapport aux valeurs précédemment rapportées. Cette chute volumétrique est en réalité hautement significative pour 3 des porcs observés (porcs 1,6 et 9) et non significative pour 3 autres animaux (porcs 4, 7 et 8). Il est intéressant de souligner que pour ces 3 derniers la durée d'interruption du recyclage entérohépatique n'a été que de 2 ou 3 jours, contre 5 ou 11 pour les porcs 1, 6 et 9 . Parallèlement, dans les $24 \mathrm{~h}$ qui suivent l'arrêt du recyclage entérohépatique, la quantité de sels biliaires exportés quotidiennement n'est plus que de $8,4 \pm 1,0 \mathrm{~g}$ (diminution de $80 \mathrm{p} .100$ par rapport à l'excrétion observée lors d'un recyclage normal de la bile). Pour tous les animaux, cette diminution est hautement significative $(P<0,001$ ou $P<0,005)$. Les valeurs enregistrées sur toute la période d'interruption ne sont pas significativement différentes, tant pour les quantités de sels biliaires que pour les volumes de bile (fig. 1 et 2), ce qui met en évidence le caractère immédiat de l'effet dépressif d'une interruption du recyclage entérohépatique sur le niveau de l'excrétion biliaire.

Enfin, le rétablissement du recyclage entérohépatique s'est accompagné d'un retour progressif (au bout de 4 à 6 jours) aux valeurs enregistrées avant l'interruption, 
ce qui traduit un bon fonctionnement des fistules mises en place (fig. 1 et 2). Par ailleurs, il n'existe apparemment aucune relation entre la durée de la dérivation biliaire et celle de la période de récupération.

Niveau de l'excrétion biliaire chez le Porc soumis à des restitutions partielles du recyclage entérohépatique.

Les résultats montrent que le niveau de l'excrétion des sels biliaires augmente avec le niveau de recyclage (tabl. 4). Les quantités de sels biliaires exportés journellement

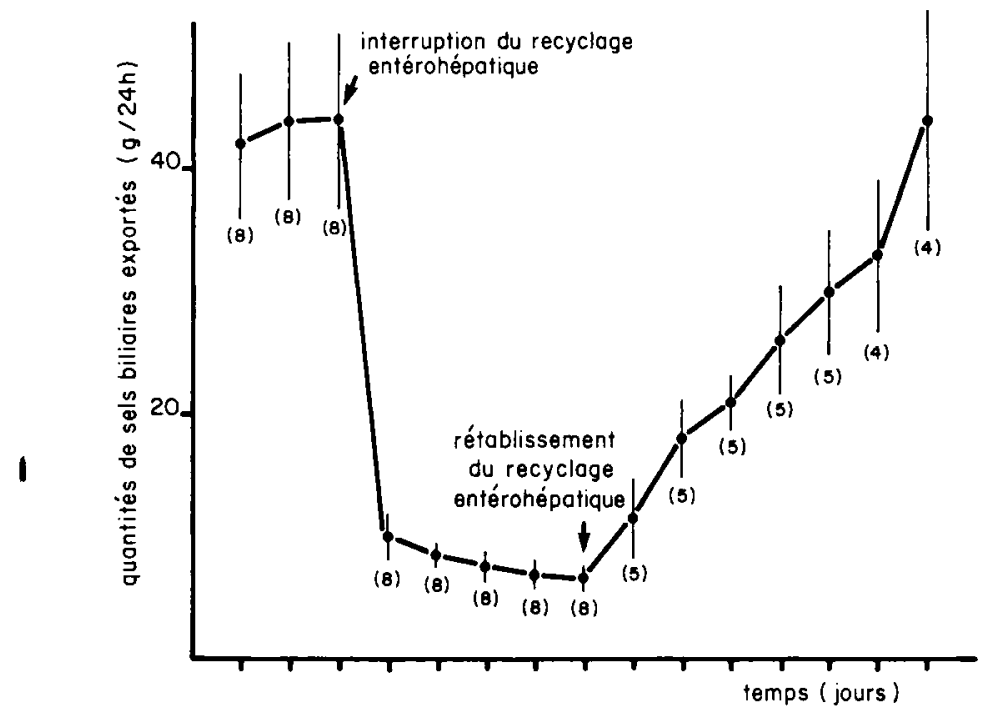

FIG. 1. - Effeł d'une interruption prolongée du recyclage entérohépatique sur la quantité de sels biliaires excrétés par $24 \mathrm{~h}$ chez le Porc. Chaque point représente la moyenne (avec l'écart-type) obtenue à partir du nombre d'animaux indiqué entre parenthèses.

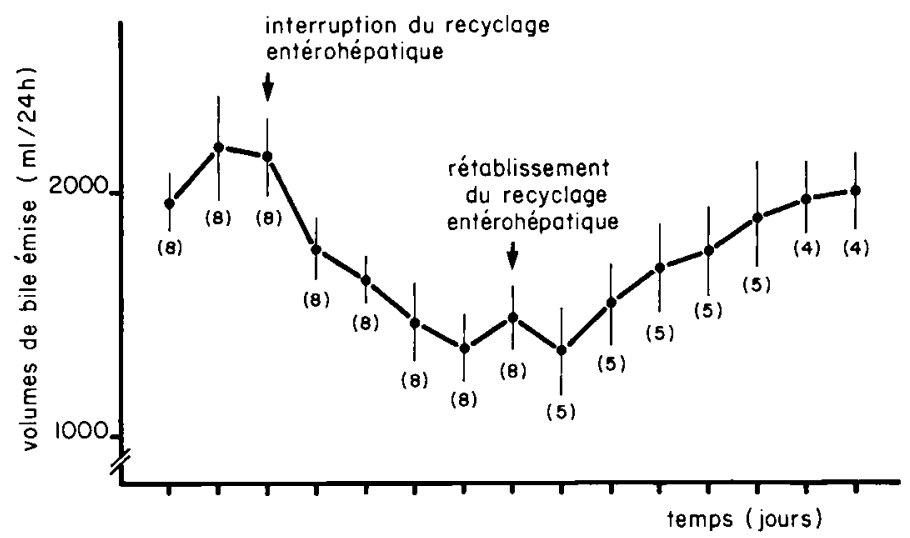

FIG. 2. - Effet d'une interruption prolongée du recyclage enférohépatique sur le volume de bile excrété par $24 \mathrm{~h}$ chez le Porc. Chaque point représente la moyenne (avec l'écart-type) obtenue à partir du nombre d'animaux indiqué entre parenthèses. 
TABLEAU 4

Effet de restitutions partielles du recyclage enféro-hépatique sur le niveou d'excréfion journalière des sels biliaires

\begin{tabular}{|c|c|c|c|c|c|}
\hline \multirow{2}{*}{$\begin{array}{l}\text { Porc } \\
1\end{array}$} & \multicolumn{2}{|c|}{$\begin{array}{l}\text { Seuil de réintroduction } \\
\text { en p. } 100^{*} \text { en } \mathrm{g} / 24 \mathrm{~h}\end{array}$} & \multicolumn{2}{|c|}{$\begin{array}{l}\text { Quantité de sels biliaires } \\
\text { excrétés par } 24 \mathrm{~h} \\
\text { en p. } 100 \quad \text { en g/ } 24 \mathrm{~h}\end{array}$} & \multirow{2}{*}{$\begin{array}{c}\begin{array}{c}\text { Nombre } \\
\text { de journées } \\
\text { d'observation }\end{array} \\
3 \\
2\end{array}$} \\
\hline & $\begin{array}{l}11 \\
25\end{array}$ & $\begin{array}{r}4,5 \\
10,2\end{array}$ & $\begin{array}{l}37 \\
47\end{array}$ & $\begin{array}{l}10,3 \pm 1,0 \\
13,1 \pm 1,0\end{array}$ & \\
\hline 2 & $\begin{array}{r}7 \\
14 \\
33\end{array}$ & $\begin{array}{r}3,1 \\
6,1 \\
14,4\end{array}$ & $\begin{array}{l}15 \\
29 \\
48\end{array}$ & $\begin{array}{r}6,5 \pm 2,5 \\
12,7 \pm 1,0 \\
21,0 \pm 2,0\end{array}$ & $\begin{array}{l}2 \\
3 \\
4\end{array}$ \\
\hline
\end{tabular}

* En p. 100 de la quantité de sels biliaires excrétés par $24 \mathrm{~h}$ dans le cas d'un recyclage total de la bile.

ont toujours été supérieures aux quantités réintroduites sur la même période (tabl. 4). Ainsi pour le Porc 2, les quantirés de sels biliaires émises en réponse aux seuils de réintroduction 7, 14 et 33 p. 100 ont été respectivement, 15, 29 et 48 p. 100 des quantités recueillies en conditions normales.

Néanmoins, aucun des seuils de recyclage biliaire, testés dans cette étude, n'a permis d'atteindre un niveau d'excrétion normal.

\section{Discussion.}

Le recyclage entérohépatique des sels biliaires a été éfudié chez le Porc, grâce à une technique de double fistulation : cholédocienne et duodénale. Cette méthode présente l'avantage de restifuer à l'animal sa propre bile, comparativement à la technique classiquement utilisée, qui consiste à maintenir artificiellement le recyclage entérohépatique, par des injections intraveineuses ou intraduodénales de sels ou acides biliaires exogènes (Eriksson, 1957; Danielsson, Einarsson et Johansson, 1967; Hoffman, Donald et Hofmann, 1977).

Interruption du recyclage entéro-hépatique.

Une interruption brutale du recyclage biliaire chez le Porc, provoque, dans les. $24 \mathrm{~h}$ qui suivent, une très forte diminution des quantités de sels biliaires excrétés, alors. que les volumes sont relativement moins affectés.

Le niveau de l'excrétion biliaire se maintient dans ce nouvel état d'équilibre pendant toute la durée de l'interruption. Il semble que la bile recueillie dans ces conditions: ne puisse provenir que d'une synthèse hépatique de novo. Cependant l'étude présente: ne permet pas de savoir, si chez le Porc, la synthèse hépatique des sels biliaires est stimulée par l'interruption du recyclage entérohépatique. Selon Dowling, Mack et Small (1970), le niveau de synthèse chez le Singe serait 4 fois supérieure à la normale, lorsque la bile est dérivée de la lumière intestinale.

Différentes espèces de laboratoire ont été soumises à des drainages permanents ef totaux de leur sécrétion biliaire. En ce qui concerne le niveau d'excrétion des sels 
biliaires dans ces conditions, les résultats apparaissent dans l'ensemble homogènes indiquant une diminution de 80 p. 100 environ des sels biliaires totaux exportés. Cette chute, également observée chez le Porc, a été rapportée chez le Chien (Whipple et Smith, 1928 ; Wheeler et Ramos, 1960), le Lapin et le Hamster (Shaw et Heath, 1974), le Mouton (Heath, Caple ef Redding, 1970), le Singe (Dowling, Mack et Swall, 1970) et le Rat (Boyer et Klatskin, 1970; Boyer, 1971).

Par contre, les volumes de bile émise en l'absence de recyclage entéro hépatique, apparaissent extrêmement variables. Chez le Chien, le Chat ef l'Homme (Wheeler et Ramos, 1970 ; Scratcherd, 1965; Thureborn, 1962), l'interruption du recyclage entérohépatique des sels biliaires entraîne une diminution importante de la quantité de bile excrétée. Par contre, chez le Rat, le Lapin et le Hamster (Klaassen, 1971, 1972 ; Shaw ef Heath, 1972, 1974 ; Scratcherd, 1965 ; Erlinger et al., 1969), une dérivation biliaire affecte peu le volume de bile émise. La présente étude montre, dans le cas du Porc, une réponse comparable à celle observée chez ces dernières espèces. Comme le suggèrent Sperber (1965), Erlinger et Preisig (1969), et Klaassen (1972), les différences observées seraient liées à l'espèce, selon l'importance des sels biliaires dans la formation de la bile. Il est donc possible que, chez le Porc, de même que chez le Rat, le Lapin et le Hamster, le transport actif d'autres solutés que les sels biliaires, soit responsable de l'importante quantité de bile excrétée en l'absence de recyclage entérohépatique. Ceci rejoindrait les résultats de Egger ef al. (1974) montrant que la formation de la bile chez le Porc, contrairement au Chien, est caractérisée par l'élaboration d'une fraction importante et indépendante des sels biliaires. Il semblerait intéressant, à ce sujet, de déterminer chez le Porc, la concentration ionique $\left(\mathrm{Cl}^{-}+\mathrm{HCO}_{3}^{-}\right)$, en l'absence de recyclage entérohépatique.

\section{Restitutions partielles de la bile recueillie.}

L'étude présente met en évidence une correspondance étroite entre le seuil de recyclage des sels biliaires recueillis après fistulation et leur niveau d'excrétion. Ceci confirme les observations réalisées après recyclage par voie veineuse ou duodénale d'un sel biliaire de nature exogène. En effet, Preisig, Cooper et Wheeler (1962) chez le Chien, Erlinger et al. (1969) chez le Lapin, Heath, Caple et Redding (1970) chez le Mouton et Egger et al. (1974) chez le Porc, montrent qu'il existe une relation positive entre les quantités de taurocholate injecté et les quantités de sels biliaires exportés.

Par ailleurs, la quantité de sels biliaires excrétés par le Porc soumis à des restitutions biliaires partielles, a toujours été supérieure à la quantité réintroduite. Mais les restitutions partielles de bile ( 7 à 33 p. 100 des sels biliaires normalement exportés) appliquées pendant 2 à 4 jours n'ont jamais permis un niveau d'excrétion normal. Ceci semblerait signifier que la compensation, probablement d'origine hépatique, est limitée. Cependant, l'éventail des restitutions partielles testées dans cette étude n'a pas permis de chiffrer cette limite. Dowling, Mack et Small (1970) montrent que le niveau de l'excrétion biliaire chez le Singe devient normal dès que le seuil de réintroduction dépasse 80 p. 100. A notre connaissance, l'origine de cette limite observée chez le Singe n'est pas expliquée. Se situe-t-elle au niveau de la synthèse du cholestérol ou des sels biliaires ou encore à un autre niveau ? 


\section{Références}

BOYER J. L., KLATSKIN G., 1970. Canalicular bile flow and bile secretory pressure. Evidence for a non-bile salt dependant fraction in the isolated rat liver. Gastroenterology, 59, 853-859.

BOYER J. L., 1971. Canalicular bile formation in the isolated perfused rat liver. Am. J. Physiol., 221, 1156-1163.

DANIELSSON H., EINARSSON K., JOHANSSON G., 1967. Effect of biliary drainage on individual reactions in the conversion of cholesterol to taurocholic acid. Europ. J. Biochem., 2, 44-49.

DOWLING R. H., MACK E., SMALL D. M., 1970. Effects of controlled interruption of the enterohepatic circulation of bile salts by biliary diversion and by ileal resection on bile salt secretion, synthesis and pool size in Rhesus monkeys. J. clin. Invest., 49, 232-242.

EGGER G., KUTZ K., STREBEL H., BICHER J., WEBER H., SCHOLL E., PREISIG R., 1974. Bile formation in the intact pig. Am. J. vet. Res., 35, 1203-1208.

ERIKSSON S., 1957. Biliary excretion of bile acids and cholesterol in bile fistula rats. Proc. Soc. exp. Biol. Med., 94, 578-582.

ERLINGER S., DHUMEAUX D., BENHAMOU J. P., FAUVERT R., 1969. La sécrétion biliaire du Lapin : preuves en faveur d'une importante fraction indépendante des sels biliaires. Rev. franç. Et. clin. biol., 14, 144-150.

ERLINGER S., PREISIG R., 1969. Les mécanismes de la cholérèse. Rev. franç. Et. clin. biol., 14,117.

HARDISON W. G. M., ROSENBERG I. H., 1967. Bile salts deficiency in the steatorrhea following resection of the ileum and proximal colon. N. Engl. J. Med., 277, 337-342.

HEATH T., CAPLE I. W., REDDING P. M., 1970. Effect of the enterohepatic circulation of bile salts on the flow of bile and its content of bile salts and lipids in sheep. Quart. J. exp. Physiol., 55, 93-103.

HOFFMAN N. E., DONALD D. E., HOFMANN A. F., 1977. Increased bile acid synthesis after interruption of the enterohepatic circulation. Evidence against hormonal mediation. Proc. Soc. exp. Biol. Med., 154, 49-52.

HOFMANN A. F., POLEY J. R., 1972. Role of bile Acid malabsorption in pathogenesis of diarrhea and steatorrhea in patients with ileal resection. Gostroenterology, 62, 918-934.

JUSTE C., CORRING T., BREANT P., 1979. Excrétion biliaire chez le Porc. Niveau et réponse au repas. Ann. Biol. anim. Bioch. Biophys., 19, 79-90.

KLAASSEN C. D., 1971. Does bile acid secretion determine canalicular bile production in rats ? Am. J. Physiol., 220, 667-673.

KLAASSEN C. D., 1972. Species differences in the choleretic response to bile salts. J. Physiol., 223, 259-269.

MC LEOD G. M., WIGGINS H. S., 1968. Bile salts in small intestinal contents after ileal resection and in other malabsorption syndromes. Lancet, 1, 873-876.

MEIHOFF W. E., KERN F., 1968. Bile salt malabsorption in regional ileitis, ileal resection and mannitol-induced diarrhoea. J. clin. Invest., 47, 261-267.

PREISIG R., COOPER H. L., WHEELER H. O., 1962. The relationship between taurocholate secretion rate and bile production in the unanesthetized dog during cholinergic blockade and during secretin administration. J. clin. Invest., 41, 1152-1162.

SCRATCHERD T., 1965. Electrolyte composition and control of biliary secretion in the cat and rabbit, 515-529. In TAYLOR W., The biliary system, Oxford, Blackwell.

SHAW H. M., HEATH T. J., 1972. The significance of hormones, bile salts and feeding in the regulation of bile and other digestive secretions in the rat. Aust. J. biol. Sci., 25, 147-154.

SHAW H. M., HEATH T. J., 1974. Regulation of bile formation in rabbits and guinea pigs. Quart. J. exp. Physiol., 59, 93-102.

SPERBER I., 1965. Biliary excretion of organic anions and its influence on bile flow, 457-467. 5 In TAYLOR W., The biliary system, Oxford, Blackwell.

THUREBORN E., 1962. Human hepatic bile. Composition changes due to altered enterohepatic circulation. Acta chir. scand., Suppl., 303, 1-63.

WHEELER H. D., RAMOS O. L., 1960 . Determinants of the flow and composition of bile in the unanesthetized dog during constant infusions of sodium taurocholate. J. clin. Invest., 39, 161-169.

WHIPPLE G. H., SMITH H. P., 1928. Bile salt metabolism. IV. How much bile salt circulates in the body? J. biol. Chem., 80, 697-707. 